Anaesthesist 2012 - 61:285-287

DOI 10.1007/s00101-012-2012-6

Online publiziert: 21. April 2012

(c) Springer-Verlag 2012

\section{P. Kranke}

Klinik und Poliklinik für Anästhesiologie, Universitätsklinikum

Würzburg, Zentrum Operative Medizin, Würzburg

\title{
„Männer sind vom Mars, Frauen von der Venus"
}

\section{"Gender medicine" als künftiges Muss auch in der Anästhesie?}

Titeln wie Männer sind vom Mars, Frauen von der Venus oder Männer sind anders. Frauen auch ist seit Jahren ein Erfolg beschieden, der seinesgleichen sucht. Parallel dazu taucht der Begriff ,gender mainstreaming“ im soziopolitischen Bereich immer häufiger auf. Das Bundesministerium für Frauen, Familie, Senioren und Jugend versteht darunter:

... bei allen gesellschaftlichen Vorhaben die unterschiedlichen Lebenssituationen und Interessen von Frauen und Männern von vornherein und regelmäßig zu berücksichtigen, da es keine geschlechtsneutrale Wirklichkeit gibt. (http://www.gender-mainstreaming.net/)

Was ist dran an der Suche nach Unterschieden, wo doch so lange stets Gleichheit, Austauschbarkeit und Vergleichbarkeit im Hinblick auf die Geschlechter betont wurden? Sollen Patienten - gerade im Hinblick auf ihr Geschlecht - womöglich doch nicht alle gleich behandelt werden, sondern müssen sie vielleicht sogar anders behandelt werden, um am Ende gleich gut behandelt zu sein?

Was in der populären Literatur seit Jahren der Hit und in der Gleichstellungspolitik der Europäischen Union seit Ende der 1990er Jahre Thema ist, kommt nun auch in der Medizin an und dies über die Fächergrenzen hinweg. Zumindest kann man sich dieses Eindrucks nicht erwehren, wenn man sich den „hype“ um „gender medicine" und individualisierte Medizin vor Augen führt. Welche Gegebenheiten gilt es zu berücksichtigen, wenn Frauen und Männer gleichermaßen gut be- handeln werden sollen? Vor diesem Hintergrund geben die Autoren Schopper et al. im Rahmen des Themenschwerpunkts der vorliegenden Ausgabe von Der Anaesthesist Antworten auf Fragen, die sonst allenfalls als Nebenaspekt, z. B. im Zuge der Pathophysiologie, der Pharmakodynamik bzw. Pharmakokinetik oder in Lehrbüchern der medizinischen Psychologie Erwähnung finden.

\section{Männer und Frauen sind gleich(berechtigt) - aber nicht in der Medizin?}

Denn auch im anästhesiologischen Erfahrungshorizont finden sich mannigfaltige Beispiele, die nahelegen, dass eben nicht alles gleich ist und Unterschiede in der Prävalenz von Erkrankungen oder der Inzidenz von Nebenwirkungen und Komplikationen sehr wohl an der Tagesordnung sind. Denken Sie an das überwiegend bei Frauen auftretende Fibromyalgiesyndrom oder den „Cluster“-Kopfschmerz, der wiederum fast nur Männer betrifft [1].

Auch unerwünschte Arzneimittelwirkungen treten oft bei einem Geschlecht gehäuft auf [2]. Und es nimmt nicht wunder, wenn die Wirkung von Opioidanalgetika nicht losgelöst von geschlechtlichen Einflüssen zu beurteilen ist [3].

Reflektiert man die diesbezügliche eigene Herangehensweise, fällt womöglich allenfalls die unterschiedliche Wahl der a priori präferierten Tubusgröße oder der Durchmesser des transurethralen Dauerkatheters auf.

Dabei - so suggeriert es die einschlägige Literatur - können die Unterschiede immens sein. Neben physiologischen Unterschieden, die oftmals durch den Einfluss der Steroidhormone erklärbar sind, ist das Geschlecht beispielsweise der am stärksten ausgeprägte prädisponierende Faktor für das Auftreten von Übelkeit und Erbrechen nach Narkosen („postoperative nausea and vomiting“, PONV). Kein anderer Prädiktor - übrigens auch das stattgehabte Auftreten von PONV bei Vornarkosen nicht - ist besser in der Lage, in einer gemischten Population eine Aussage darüber zu treffen, ob nach einer Allgemeinanästhesie mit PONV zu rechnen ist oder nicht. Folglich wäre es geradezu konsequent, allen Frauen ein wenig mehr antiemetische Prophylaxe zukommen zu lassen, wenn man denn überhaupt ein abgestuftes Vorgehen für sinnvoll erachtet und nicht einer generellen multimodalen Prophylaxe den Vorzug gibt $[4,5]$.

\section{Notfall: Eilt es bei Frauen weniger?}

Und selbst in der Notfallmedizin lohnt offenkundig ein Blick auf die tatsächlich gegebene Versorgungsrealität und mögliche Einflussfaktoren. So weisen jüngst publizierte Daten darauf hin, dass nach

Gray J (1999) Jeden Tag mehr Liebe - Männer sind vom Mars, Frauen von der Venus. Goldmann, München. 
Implementierung einer prähospitalen EKG-Analyse im Kontext des Meldebilds "chest pain“ die Zeit bis zur Krankenhausaufnahme nicht zwingend länger dauern muss, dass aber bei Betrachtung des Geschlechts als Einflussfaktor zeitliche Differenzen - im Sinne einer verlängerten prähospitalen Zeit bei Frauen - auftreten [6], die schlussendlich durchaus (negative) Auswirkungen auf eine zeitgerechte Versorgung und konsekutiv das „outcome“, z. B. bei akutem Koronarsyndrom, haben könnten. Diese Zahlen - so sie sich bestätigen lassen - können als Hinweis auf eine unterschiedliche Versorgungsrealität interpretiert werden. Dabei muss derzeit offen bleiben, ob die Einstellungen des medizinischen Personals oder eben schlicht und ergreifend eine andere Priorisierung seitens der betroffenen $\mathrm{Pa}$ tientinnen im Zuge der Berücksichtigung von Patienteninteressen (Stichwort: „shared decision-making " und partizipative Entscheidungsfindung) hierfür maßgeblich gewesen ist. Die Frage nach der Koexistenz oder einer möglichen Kausalität schwingt hier ebenso mit wie die Frage nach der Definition einer "guten Behandlung “. Mitnichten wurde bislang ein Überlebensvorteil in diesem Kontext gezeigt, ganz zu schweigen davon analysiert, wie gut der Patientenwunsch Berücksichtigung fand.

Oft erscheint die Unterscheidung, was dem Gender-Effekt und damit genetisch determinierten Unterschieden auf der einen Seite und was dem Gender-Bias also der unwillkürlich anders erfolgenden Versorgung bei Patienten unterschiedlichen Geschlechts - auf der anderen Seite „anzulasten“ ist, noch nebulös und einer weiteren Untersuchung bedürftig.

Da sich allerdings populärwissenschaftliche Veröffentlichungen mit groBem Erfolg dieses Themas annehmen bzw. Spekulationen dazu anstellen, ist die seriöse wissenschaftliche Auseinandersetzung für uns umso bedeutsamer.

\section{Genaues Hinsehen bei allen Patienten ist nötig}

Schopper et al. zeigen in ihrem Beitrag auf, wie umfassend das Thema Gender medicine mit allen seinen Facetten bereits jetzt ist und welche Aspekte schon zum gegen- wärtigen Zeitpunkt Berücksichtigung in der klinischen Versorgungsrealität finden könnten - auch in der Anästhesiologie.

Dabei steht die genaue Ursachendetektion und -analyse - obgleich wünschenswert - nicht einmal im Vordergrund, gilt es doch vor dem Hintergrund des Patientenwunsches zunächst nur, eine gute Versorgung sicherzustellen. Allerdings wäre es gefährlich, Erkenntnisse wie z. B. die größere Gefahr durch Medikamentennebenwirkungen oder das höhere Risiko einer Nebenwirkung nach bestimmten Interventionen nicht zu beachten, weil man die soziokulturelle Diskussion für überflüssig hält. Auffällige Unterschiede auch in eigenen Daten, z. B. im Rahmen eines Qualitätsmanagements, sollten folglich Anlass zur kritischen Reflexion des eigenen Vorgehens und Grund genug dafür sein, die Versorgungsrealität für Subpopulationen auf den Prüfstand zu stellen.

Die Polarisierung gemäß dem Geschlecht oder anderen phänotypisch auffälligen Eigenschaften der Patienten greift mittelfristig sicher zu kurz und kann nur ein kleiner Teilaspekt im Bestreben hin zu einer individualisierten Medizin sein. Ein Blick auf die landauf, landab verbreiteten Vorurteile mit Fokus auf die Hautund Haarfarbe sowie die Empfindlichkeit gegenüber Analgetika und Anästhetika zeigt jedoch, wie stark sie in der allgemeinen Wahrnehmung verwurzelt ist. Häufig gelten rothaarige Frauen als besonders wehleidig. Tatsächlich scheint beispielsweise das an $\kappa$-Opioid-Rezeptoren bindende Pentazocin bei Frauen in Abhängigkeit von der Variante des Gens Mclr gut oder weniger gut zu wirken; der „melanocortin 1 receptor“ (Mclr) sorgt offenbar nicht nur für die Produktion des Pigments Phäomelanin bei rothaarigen Menschen, sondern beeinflusst auch die Schmerzwahrnehmung und das Ansprechen auf bestimmte Analgetika.

Eine leicht $\mathrm{zu}$ verallgemeinernde Schlussfolgerung ist hier aber sowohl im Hinblick auf die individuelle Reaktion auf schmerzhafte Stimuli und damit einhergehend die Angst vor potenziell schmerzhafter Behandlung [7] ebenso wenig abzugeben, wie eine generelle Aussage bezüglich des Ansprechens auf Anästhetika [8] und Analgetika $[9,10]$ bzw. der mit ihrer Applikation assoziierten Nebenwirkun- gen. Vielmehr muss sorgfältig nach Evidenz gesucht und somit gewissermaßen die populäre „Spreu“ vom wissenschaftlichen „Weizen“ getrennt werden.

Folglich implizieren Gender medicine und der Fokus darauf in einer globaleren Betrachtungsweise aus Sicht des Autors die Aufforderung, genau hinzusehen, welche geschlechtsbezogenen Ursachen oder pharmakogenetisch relevanten Variablen einer unterschiedliche Prävalenz oder Disposition zugrunde liegen. Nur so wird es gelingen, Stück für Stück (Stichwort: individualisierte Behandlung bzw. Therapie) den Bedürfnissen des einzelnen Patienten besser gerecht zu werden als bisher. Dabei sollte es primär unerheblich sein, ob der Einflussfaktor nun im Geschlecht in engerem Sinn, in einer spezifischen Enzym- oder Genausstattung bei gleichem Geschlecht oder im "Gender“ im Sinne der eingangs erwähnten umfassenderen Definition - liegt. In vielen klinischen Bereichen ist eine derartige Individualisierung der Therapie bereits grundsätzlich denkbar [11, 12]. Auch wenn die baldige breite Implementierung noch Wunschgedanke sein mag [13], werden auf absehbare Zeit derlei Untersuchungen sicher dazu beitragen, klinische Erfahrungen und bewiesene Assoziationen, z. B. bezüglich geschlechtsbezogener Effekte erklärbar zu machen.

Und wenn in der englischen Ausgabe von Grays "Men are from Mars, women are from Venus" als Untertitel zu lesen ist: "A Practical Guide for Improving Communication and Getting What You Want in Your Relationships", dann könnte - bezogen auf Gender medicine - die erläuternde Bemerkung und assoziierte Forderung sein: Forschung betreiben, um $\mathrm{Ge}$ schlechtsunterschiede zu erkennen und die Erkenntnisse in der klinischen $\mathrm{Me}$ dizin/Pharmakotherapie zur Optimierung von Wirkung und Minimierung von Nebenwirkungen zielgerecht einsetzen.

Ein Umstand, der möglicherweise Ihren Patienten bereits bewusst ist, wenn diese sich in einschlägigen Foren oder auf Webseiten zur Gender-Forschung informiert oder schlicht und ergreifend erneut in das Regal mit der populärwissenschaftlichen Literatur gegriffen haben und den Titel Frauen sind anders krank als Männer - Plädoyer für eine geschlechtsspezifische 
Medizin [14] gelesen haben. Seien Sie also gewappnet mit dem neuesten Erkenntnissen zu anästhesiologischen Aspekten von Gender medicine, den geschlechtsspezifischen Prävalenzen für Erkrankungen, der unterschiedlichen Inzidenz von Nebenwirkung oder der variablen „response auf Ihre Pharmakotherapie, die mitunter eben mehr ist als eine unterschiedliche „Wehleidigkeit“ oder vielleicht der Drang unserer Patienten, den ,tapferen Indianer“ zu mimen.

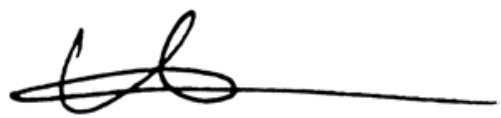

Peter Kranke

\section{Korrespondenzadresse}

\section{Prof. Dr. P. Kranke}

Klinik und Poliklinik für Anästhesiologie, Universitätsklinikum Würzburg, Zentrum Operative Medizin

Oberdürrbacher Str. 6, 97080 Würzburg

kranke_p@klinik.uni-wuerzburg.de

Interessenkonflikt. Der korrespondierende Autor gibt an, dass kein Interessenkonflikt besteht.

\section{Literatur}

1. Greenspan JD, Craft RM, LeResche L et al. (2007) Studying sex and gender differences in pain and analgesia: a consensus report. Pain 132 (Suppl 1):26-45

2. Soldin OP, Chung SH, Mattison DR (2011) Sex differences in drug disposition. J Biomed Biotechnol. DOI 10.1155/2011/187103

3. Niesters M, Dahan A, Kest B et al (2010) Do sex differences exist in opioid analgesia? A systematic review and meta-analysis of human experimental and clinical studies. Pain 151:61-68

4. Kranke P (2011) Effective management of postoperative nausea and vomiting: let us practise what we preach! Eur J Anaesthesiol 28:152-154

5. Kranke P, Eberhart LH (2011) Possibilities and limitations in the pharmacological management of postoperative nausea and vomiting. Eur J Anaesthesiol 28:758-765

6. Aguilar SA, Patel M, Castillo E, et al (2012) Gender differences in scene time, transport time, and total scene to hospital arrival time determined by the use of a prehospital electrocardiogram in patients with complaint of chest pain. J Emerg Med. DOI http://dx.doi.org/10.1016/j.jemermed.2011.06.130

7. Binkley CJ, Beacham A, Neace W et al (2009) Genetic variations associated with red hair color and fear of dental pain, anxiety regarding dental care and avoidance of dental care. J Am Dent Assoc 140:896-905
8. Liem EB, Lin CM, Suleman Ml et al (2004) Anesthetic requirement is increased in redheads. Anesthesiology 101:279-283

9. Mogil JS, Wilson SG, Chesler EJ et al (2003) The melanocortin-1 receptor gene mediates femalespecific mechanisms of analgesia in mice and humans. Proc Natl Acad Sci U S A 100:4867-4872

10. Mogil JS, Ritchie J, Smith SB et al (2005) Melanocortin-1 receptor gene variants affect pain and mu-opioid analgesia in mice and humans. J Med Genet 42:583-587

11. Mega JL, Hochholzer W, Frelinger AL 3rd et a (2011) Dosing clopidogrel based on CYP2C19 genotype and the effect on platelet reactivity in patients with stable cardiovascular disease. JAMA 306:2221-2228

12. Candiotti KA, Birnbach DJ, Lubarsky DA et al (2005) The impact of pharmacogenomics on postoperative nausea and vomiting: do CYP2D6 allele copy number and polymorphisms affect the success or failure of ondansetron prophylaxis? Anesthesiology 102:543-549

13. Candiotti K (2009) Anesthesia and pharmacogenomics: not ready for prime time. Anesth Analg 109:1377-1378

14. Voß A (2007) Frauen sind anders krank als Männer - Plädoyer für eine geschlechtsspezifische Medizin. Irisana, München

\section{Aktionsbündis Patienten- sicherheit}

Das seit 2005 bestehende Aktionsbündnis

Patientensicherheit e.V. hat sich zur Aufgabe gemacht, die Patientensicherheit in Deutschland zu fördern und die Erforschung, Entwicklung und Verbreitung dazu geeigneter Methoden zu unterstützen. Konkret heißt das, eine Schädigung der Patienten im Behandlungs- und Versorgungsprozess zu vermeiden und auf mögliche Risiken aufmerksam zu machen. Die Zusammenarbeit dient dem fachlichen Austausch, sowie der Vorbereitung und Durchführung von Aktionen und Kampagnen zur Verbesserung der Patientensicherheit. Laufende Projekte sind hierbei beispielsweise, die „Aktion Saubere Hände“, die zu einer verbesserten Aufmerksamkeit bei der Händedesinfektion führen soll.

Ebenso das Krankenhaus-CIRS-Netzwerk (Critical Incident Reporting System), das vermehrt in deutschen Krankenhäusern eingeführt werden soll. Weiterhin sollen Probleme im klinischen Alltag mehr in den Fokus gelegt werden. Dabei sind die Vermeidung unbeabsichtigt belassener Fremdkörper im OP-Gebiet oder Eingriffsverwechslungen in der Chirurgie zu erwähnen. Das Aktionsbündnis Patientensicherheit pflegt Kooperationen mit Verbänden, Fachgesellschaften, Forschungsinstituten, Krankenkassen und Patientenorganisationen und wird vom Bundesministerium für Gesundheit unterstützt. Der Verein wirbt aktiv um Mitglieder und heißt Spenden willkommen.

Quelle:

Aktionsbündnis Patientensicherheit e.V., www.aps-ev.de 